\title{
Preexisting Neutralizing Antibodies to Adeno-Associated Virus Capsids in Large Animals Other Than Monkeys May Confound In Vivo Gene Therapy Studies
}

\author{
Roberto Calcedo,' Judith Franco,' Qiuyue Qin,, Dean W. Richardson, Jeffery B. Mason,2 \\ Surina Boyd, and James M. Wilson ${ }^{1}$
}

\begin{abstract}
Adeno-associated virus (AAV) vectors are currently being tested not only in small animal models such as mice but also in large animal models, including pigs, dogs, and horses. Natural exposure to AAV occurs in most of the species used in these studies and potentially elicits a neutralizing humoral immune response to AAV. In this study, we show the prevalence of neutralizing antibodies (NAbs) to several AAV serotypes in these large animals as measured by an in vitro NAb assay and the ability of these NAbs to block AAV transduction in an in vivo mouse model of NAb passive transfer assay. The results of this study clearly show the importance of evaluating large animal models for the presence of AAV NAbs before enrolling them in AAV-mediated gene therapy studies.
\end{abstract}

\section{Introduction}

T IS IMPORTANT FOR THE SUCCESS of gene therapy to use animal models other than mice, which can give us a better understanding of the performance and potential toxicity of adeno-associated virus (AAV) vectors before they are used in human clinical trials. One aspect that affects toxicity and in vivo vector performance is natural exposure to viruses that are similar to the virus used to create the vector. This can lead to the induction of a neutralizing humoral immune response. AAV is a virus that belongs to the parvovirus family, which causes natural infections in many species including humans, monkeys, pigs, dogs, and horses potentially inducing B-cell responses to the virus. We and others have demonstrated that low levels of preexisting NAb to AAV vectors in monkeys have a profound impact on gene transfer ${ }^{1-3}$ and redistribution of the vector to other organs such as the spleen. ${ }^{4}$ The natural presence of AAV NAbs was not considered to have an impact in nonprimate animal models because their natural AAVs were thought to be serologically different from primate AAVs. A study was conducted to demonstrate this hypothesis. We selected AAV capsids used in gene therapy preclinical studies (AAV1, AAV2, AAV5, AAV6, and AAV9). These five AAV capsids were evaluated for the presence of AAV NAbs in serum from horses, dogs, and pigs, which are used as preclinical models for human diseases. Horse is the primary model for osteoarthritis, ${ }^{5}$ while dog is the primary model for Duchene muscular dystrophy (DMD) and FIX deficiency, ${ }^{6-8}$ and pig is the model for several heartrelated gene therapies. 9,10

\section{Materials and Methods}

Vector construction, production, and purification: AAV1, AAV2, AAV5, AAV6, and AAV8 recombinant vectors used in this study were synthesized and purified as previously described by the Penn Vector Core at the University of Pennsylvania. ${ }^{11,12}$ Each AAV serotype was constructed expressing $\beta$-galacotsiadase (LacZ) or canine FIX (cFIX) controlled by a cytomegalovirus (CMV) promoter or by the liver-specific thyroid hormonebinding globulin promoter (TBG), respectively. ${ }^{11,12}$ Titration of AAV vector genomes (genome copies/ml) was quantified by real-time PCR using a primer/probe set corresponding to the polyA region of the vector and linearized plasmid standards.

Serum samples: Horse serum samples $(n=12)$ were obtained from New Bolton Center (University of Pennsylvania, PA); dog serum samples $(n=70)$ were obtained from the School of Veterinary Medicine (University of Pennsylvania); and pig serum samples $(n=17)$ were obtained from Case Western Reserve University. Serum samples for the different species were heat inactivated at $56^{\circ} \mathrm{C}$ for $35 \mathrm{~min}$ and archived at $-20^{\circ} \mathrm{C}$ for later NAb analysis.

In vitro neutralizing antibody assay: Heat inactivated serum samples from the different species were evaluated for the presence of neutralizing antibodies as previously described. ${ }^{13}$ Limit of detection of the assay was $1 / 5$ serum dilution.

\footnotetext{
${ }^{1}$ Department of Pathology and Laboratory Medicine, School of Medicine and Department of Clinical Studies; ${ }^{2}$ New Bolton Center, School of Veterinary Medicine; University of Pennsylvania, Philadelphia, PA 19104.
} 

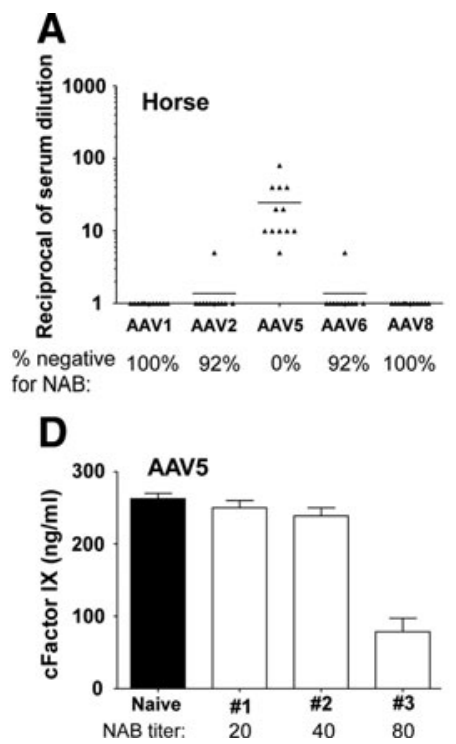
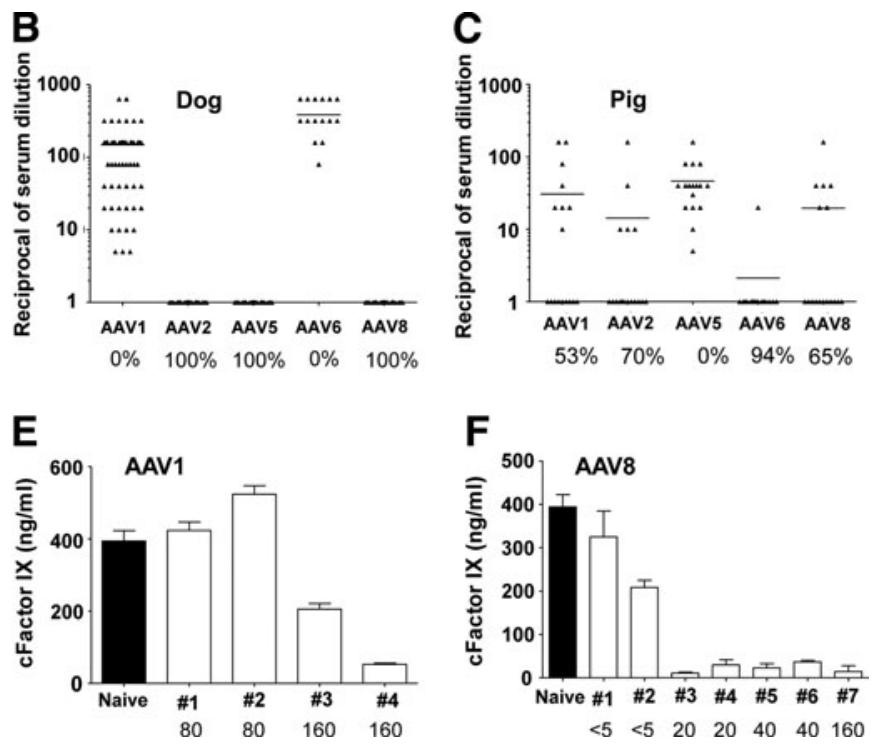

FIG. 1. Detection of adeno-associated virus (AAV) neutralizing antibodies (NAbs). Prevalence of NAbs against various AAV types in serum samples from horses (A), dogs (B), and pigs (C) as measured by in vitro NAb assay. The interference of $\mathrm{NAb}$ in AAV-mediated gene transfer was studied in C57BL/6 mice injected with $3 \times 10^{10}$ GC of AAV5 (D), AAV1 (E), or $1 \times 10^{9} \mathrm{GC}$ of AAV8.LSP.cFIX (F) after having received serum from horses (D), dogs (E), and pigs (F) with different NAb titers. Canine FIX expression was measured in plasma 7 days after vector administration.

In vivo neutralizing antibody assay: Heat inactivated serum samples and AAV vectors were administered to C57BL/6 mice and FIX levels measured as previously described. ${ }^{12}$ AAV8 was injected to a dose of $10^{9} \mathrm{GC} /$ mouse and AAV1 and AAV5 to a dose of $3 \times 10^{10} \mathrm{GC} /$ mouse.

\section{Results and Discussion}

A total of 99 serum samples from large animals were evaluated for the presence of AAV NAbs by an in vitro transduction inhibition assay. Interestingly, a large number of animals were positive for AAV NAbs. This elevated seroprevalence was serotype and species specific.

In horses (Fig. 1A), AAV5 was the dominant AAV serotype with all the samples testing positive for NAbs. We detected low or no NAb to other AAV serotypes. In dogs (Fig. 1B), AAV serotypes 1 and 6 were the dominant AAVs, with all the samples positive for NAbs; we did not detect the presence of NAbs in other AAV serotypes. No discrepancies in AAV seroprevalence were found when dogs from a different colony and genetic background ${ }^{14}$ were analyzed. In pigs (Fig. 1C), we found that AAV5 again was the dominant AAV serotype with all the samples positive for NAbs. The presence of NAbs to the other AAV serotypes was more diverse and ranged from 35 to $47 \%$. In this species the serotype least seroprevalent was AAV6, with only $6 \%$ of the samples positive for NAbs.

A number of serum samples from the species described above that tested positive for the presence of AAV NAbs using the in vitro transduction inhibition NAb assay were evaluated using an in vivo mouse model of NAb assay. In this in vivo $\mathrm{NAb}$ assay, individual serum samples from all three animal species were injected into mice before systemic administration of an AAV vector was synthesized, with the capsid of interest as follows: AAV5 for horses (Fig. 1D), AAV1 for dogs (Fig. 1E), and AAV8 for pigs (Fig. 1F).
Mice were injected with the minimum dose that achieved a detectable FIX expression in plasma: $3 \times 10^{10} \mathrm{GC}$ for AAV1 and AAV5 and $1 \times 10^{9} \mathrm{GC}$ for AAV8 (Fig. 2).

In vivo gene transfer by $\mathrm{AAV}$ in passively immunized mice was abrogated or significantly reduced after systemic administration of the vector by serum samples that identified as positive for AAV NAbs by the in vitro transduction inhibition assay confirming the functionality of the in vitro detected NAbs. On AAV8 injected animals, a NAb titer of 1/ 20 was enough to completely block transduction. Our results agree with previous published data in which systemic administration of AAV8 vectors in monkeys with a preexisting

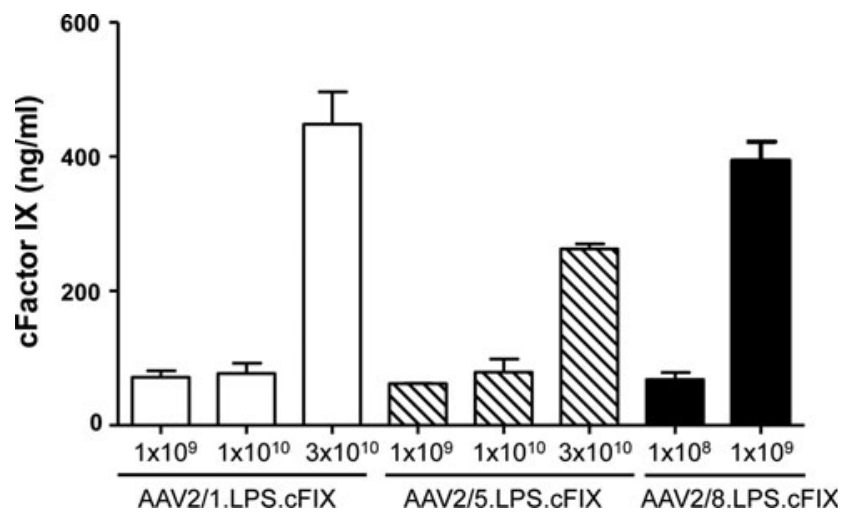

FIG. 2. Minimum AAV vector dose needed for cFIX transgene detection in plasma. AAV2/1, AA2/5, and AAV2/ 8.LSP.cFIX vectors were administered intramuscularly to three C57BL/6 mice per vector dose, measured as genome copies, and cFIX levels in plasma measured 7 days after vector administration. The vector dose that yields levels of cFIX in plasma of at least $200 \mathrm{ng} / \mathrm{ml}$ was selected for the in vivo neutralizing antibody assay. 
AAV8 NAb titer greater than 10 was enough to significantly reduce transduction of the liver. ${ }^{4}$ On AAV1- and AAV5injected animals a NAb titer of $1 / 160$ and 1/80 respectively was enough to significantly inhibit transduction.

Differences in NAb titer needed for a significant reduction of in vivo transduction can be attributed to the vector dose injected in each animal group, which was 30-fold lower in the AAV8 group. These data show the important correlation between vector dose, preexisting AAV NAb titer, and transduction level.

The data generated in this study highlights the importance of evaluating all animal species for the presence of natural NAbs to AAV when designing AAV-mediated gene transfer studies and evaluating the effect that preexisting $\mathrm{NAb}$ and vector doses may have on in vivo transduction and host immune responses to AAV vectors.

\section{Acknowledgments}

We would like to thank Kevin Donahue (Case Western Reserve University) for the pig serum samples and Mark Haskins (School of Veterinary Medicine, University of Pennsylvania) for the dog serum samples. This work was funded by a grant from the National Institute of Diabetes and Digestive and Kidney Diseases (NIDDK), P30DK047757 (JMW), and a grant from Grayson Jockey Club Foundation (DWR).

\section{Author Disclosure Statement}

James M. Wilson is an advisor to REGENXBIO, Dimension Therapeutics, Solid Gene Therapy, and Alexion, and is a founder of, holds equity in, and has a sponsored research agreement with REGENXBIO and Dimension Therapeutics. In addition, he is a consultant to several biopharmaceutical companies and is an inventor on patents licensed to various biopharmaceutical companies. The rest of the coauthors have no conflicts of interest to disclose.

\section{References}

1. Scallan CD, Jiang H, Liu T, et al. Human immunoglobulin inhibits liver transduction by AAV vectors at low AAV2 neutralizing titers in SCID mice. Blood 2006;107:18101817.

2. Hurlbut GD, Ziegler RJ, Nietupski JB, et al. Preexisting immunity and low expression in primates highlight translational challenges for liver-directed AAV8-mediated gene therapy. Mol Ther 18:1983-1994.

3. Jiang H, Couto LB, Patarroyo-White S, et al. Effects of transient immunosuppression on adenoassociated, virusmediated, liver-directed gene transfer in rhesus macaques and implications for human gene therapy. Blood 2006;108: 3321-3328.
4. Wang L, Calcedo R, Bell P, Lin J, Grant RL, Siegel DL, Wilson JM. Impact of pre-existing immunity of gene transfer to nonhuman primate liver with AAV8. Hum Gen Ther 2011;22:1389-1401.

5. Frisbie DD, McIlwraith CW. Evaluation of gene therapy as a treatment for equine traumatic arthritis and osteoarthritis. Clin Orthop Relat Res 2000(379 Suppl):S273-287.

6. Wang Z, Kuhr CS, Allen JM, et al. Sustained AAV-mediated dystrophin expression in a canine model of Duchenne muscular dystrophy with a brief course of immunosuppression. Mol Ther 2007;15:1160-1166.

7. Yuasa K, Yoshimura M, Urasawa N, et al. Injection of a recombinant AAV serotype 2 into canine skeletal muscles evokes strong immune responses against transgene products. Gene Ther 2007;14:1249-1260.

8. Wang L, Nichols TC, Read MS, et al. Sustained expression of therapeutic level of factor IX in hemophilia B dogs by AAV-mediated gene therapy in liver. Mol Ther 2000;1: 154-158.

9. Tao Z, Chen B, Tan X, et al. Coexpression of VEGF and angiopoietin-1 promotes angiogenesis and cardiomyocyte proliferation reduces apoptosis in porcine myocardial infarction (MI) heart. Proc Natl Acad Sci U S A 108:2064-2069.

10. Hadri L, Bobe R, Kawase Y, et al. SERCA2a gene transfer enhances eNOS expression and activity in endothelial cells. Mol Ther 18:1284-1292.

11. Gao G, Lu Y, Calcedo R, et al. Biology of AAV serotype vectors in liver-directed gene transfer to nonhuman primates. Mol Ther 2006;13:77-87.

12. Wang L, Calcedo R, Wang H, et al. The pleiotropic effects of natural AAV infections on liver-directed gene transfer in macaques. Mol Ther 18:126-134.

13. Calcedo R, Vandenberghe LH, Gao G, et al. Worldwide epidemiology of neutralizing antibodies to adeno-associated viruses. J Infect Dis 2009;199:381-390.

14. Hakim CH, Yue Y, Shin JH, et al. Systemic gene transfer reveals distinctive muscle transduction profile of tyrosine mutant AAV-1, -6 , and -9 in neonatal dogs. Mol Ther Methods Clin Dev 2014;1:14002.

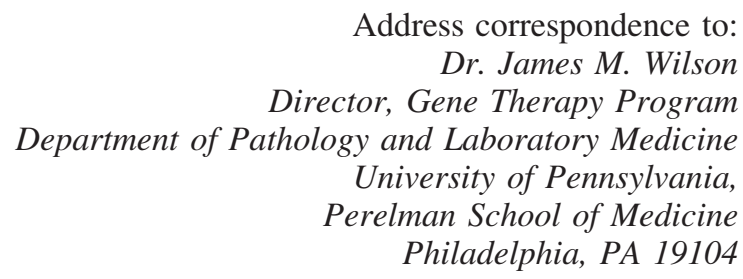

E-mail: wilsonjm@mail.med.upenn.edu

Received for publication May 29, 2015;

accepted after revision June 10, 2015.

Published online: June 10, 2015. 